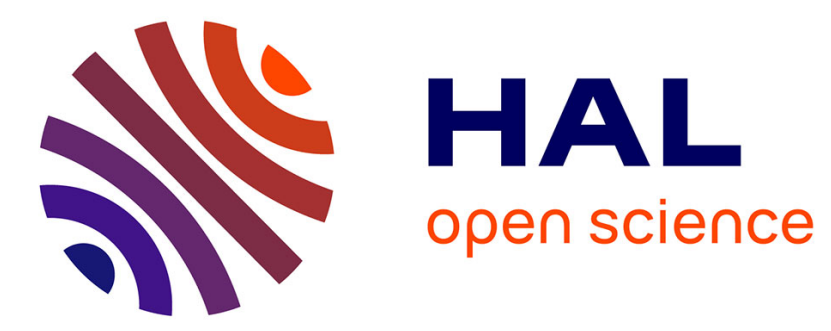

\title{
Problèmes capacitaires en viscoplasticité avec effets de torsion
}

Michel Bellieud

\section{To cite this version:}

Michel Bellieud. Problèmes capacitaires en viscoplasticité avec effets de torsion. Comptes Rendus. Mathématique, 2013, 351 (5-6), pp.241-245. 10.1016/j.crma.2013.03.005 . hal-00802121

\section{HAL Id: hal-00802121}

\section{https://hal.science/hal-00802121}

Submitted on 19 Mar 2013

HAL is a multi-disciplinary open access archive for the deposit and dissemination of scientific research documents, whether they are published or not. The documents may come from teaching and research institutions in France or abroad, or from public or private research centers.
L'archive ouverte pluridisciplinaire HAL, est destinée au dépôt et à la diffusion de documents scientifiques de niveau recherche, publiés ou non, émanant des établissements d'enseignement et de recherche français ou étrangers, des laboratoires publics ou privés. 
Note proposée aux "Comptes Rendus Mathématiques", rubrique 29 : "Problèmes Mathématiques de la Mécanique".

\title{
Problèmes capacitaires en viscoplasticité avec effets de torsion.
}

\author{
Bellieud Michel ${ }^{\mathrm{a}}$, \\ ${ }^{\mathrm{a}} \mathrm{LMGC}$, UMR-CNRS 5508, Université Montpellier II, Case courier 048, Place Eugène Bataillon, 34095 Montpellier \\ Cedex 5. \\ Reçu le ${ }^{* * * * *} ;$ accepté après révision le +++++ \\ Présenté par
}

\section{Résumé}

Nous étudions l'homogénéisation de problèmes du type

$$
\left\{\begin{array}{l}
\min _{\boldsymbol{u}_{\varepsilon} \in W_{b}^{1, p}\left(\Omega ; \mathbb{R}^{3}\right)} F_{\varepsilon}\left(\boldsymbol{u}_{\varepsilon}\right)-\int_{\Omega} \boldsymbol{f} \cdot \boldsymbol{u}_{\varepsilon} d x, \quad \boldsymbol{f} \in L^{p^{\prime}}\left(\Omega ; \mathbb{R}^{3}\right), \quad\left(\frac{1}{p}+\frac{1}{p^{\prime}}=1\right), \\
W_{b}^{1, p}\left(\Omega ; \mathbb{R}^{3}\right)=\left\{\boldsymbol{\psi} \in W^{1, p}\left(\Omega ; \mathbb{R}^{3}\right), \boldsymbol{\psi}=0 \text { sur } \widehat{\Omega} \times\{0\}\right\}, \\
F_{\varepsilon}\left(\boldsymbol{u}_{\varepsilon}\right):=\int_{\Omega \backslash T_{r_{\varepsilon}}} f\left(\boldsymbol{e}\left(\boldsymbol{u}_{\varepsilon}\right)\right) d x+k_{\varepsilon} \int_{T_{r_{\varepsilon}}} g\left(\boldsymbol{e}\left(\boldsymbol{u}_{\varepsilon}\right)\right) d x, \quad \boldsymbol{e}\left(\boldsymbol{u}_{\varepsilon}\right)=\frac{1}{2}\left(\nabla \boldsymbol{u}_{\varepsilon}+\nabla^{T} \boldsymbol{u}_{\varepsilon}\right),
\end{array}\right.
$$

lorsque $f, g$ sont des fonctions strictement convexes satisfaisant des conditions de croissance d'ordre $p \in(1,+\infty)$, $g$ est positivement homogène de degré $p, k_{\varepsilon}$ tend vers $+\infty$, et $T_{r_{\varepsilon}}$ est une distribution $\varepsilon$-périodique de fibres parallèles de section de taille $r_{\varepsilon}<<\varepsilon$. Le problème (1) correspond à un modèle simplifié d'élasticité non linéaire en petites déformations décrivant par exemple les petites déformations d'un matériau de Ogden [8]. Lorsque $1<p<2$, il est aussi employé pour décrire le fluage à haute température d'un composite métallique dans le modèle de Norton-Hoff [7]. Dans ce cas, $\boldsymbol{u}_{\varepsilon}$ représente le champ des vitesses. Nous prouvons que lorsque $p \leq 2$, une concentration d'énergie élastique apparaît dans une petite zone entourant les fibres. Elle s'exprime en fonction de la densité locale des sections des fibres relativement à une capacité spécifique tenant compte, si $p<2$, des angles de rotation des fibres par rapport à leur axe principal. Ce comportement rotatoire génère, en parallèle, une concentration d'énergie de torsion à l'intérieur des fibres.

Pour citer cet article :

Abstract

Capacitary problems in elasticity with torsion effects. We study the homogenization of elasticity problems like (1) when $f, g$ are strictly convex functions satisfying a growth condition of order $p \in(1,+\infty), g$ is positively homogeneous of degree $p, k_{\varepsilon} \rightarrow+\infty$, and $T_{r_{\varepsilon}}$ consists of an $\varepsilon$-periodic distribution of parallel fibers of cross sections of size $r_{\varepsilon}<<\varepsilon$. The problem (1) corresponds to a simplified model of small deformation nonlinear elasticity describing for instance the small deformations of an Ogden's material [8]. When $1<p<2$, it may also characterize the viscoplastic creep experienced, at high temperatures, by a metallic composite governed by the Norton-Hoff model [7]. In this case, $\boldsymbol{u}_{\varepsilon}$ represents the velocity vector field. We show that if $p \leq 2$, a concentration of strain energy appears in a small region of space surrounding the fibers. This extra contribution is characterized 
by a local density of the sections of the fibers with respect to some appropriate capacity depending, if $p<2$, on the angles of rotation of the fibers with respect to their principal axis. This rotating behavior generates, in parallel, the emergence of torsional strain energy within the fibers.

To cite this article:

\section{Abridged English version}

Let $\Omega:=\widehat{\Omega} \times(0, L)$ be a bounded cylindrical domain of $\mathbb{R}^{3}$. Given a bounded Lipschitz domain $S$ of $\mathbb{R}^{2}$ and a sequence $\left(r_{\varepsilon}\right)$ of positive reals such that $0<r_{\varepsilon}<<\varepsilon$, we denote by $T_{r_{\varepsilon}}$ the $\varepsilon$-periodic distribution of parallel "fibers" defined by (4). We are concerned with the homogenization of the elasticity problem $(1)$, where, denoting by $\mathbb{S}^{3}$ the set of all real symmetric matrices of order 3 , the strictly convex functions $f, g: \mathbb{S}^{3} \rightarrow \mathbb{R}$ satisfy a growth condition of order $p \in(1,+\infty)$ (see $(5)$ ), $g$ is positively homogeneous of degree $p$, and $\left(k_{\varepsilon}\right)$ is a sequence of positive reals verifying (6). The limiting problem is expressed in terms of the limit $\boldsymbol{u}$ of the sequence $\left(\boldsymbol{u}_{\varepsilon}\right)$ of the solutions to (1), the limit $(\boldsymbol{v}, \theta)$ of the sequence $\left(\boldsymbol{v}_{\varepsilon}\left(\boldsymbol{u}_{\varepsilon}\right), \theta_{\varepsilon}\left(\boldsymbol{u}_{\varepsilon}\right)\right)$, where the operators $\mathfrak{v}_{\varepsilon}$ and $\theta_{\varepsilon}$ are defined on $W^{1, p}\left(\Omega ; \mathbb{R}^{3}\right)$ by $(7)$ and, if $0<\kappa<+\infty$, the limit $(w, \delta)$ of the sequence $\left(\frac{1}{r_{\varepsilon}} \mathfrak{v}_{\varepsilon 3}\left(\boldsymbol{u}_{\varepsilon}\right), \frac{1}{r_{\varepsilon}} \theta_{\varepsilon}\left(\boldsymbol{u}_{\varepsilon}\right)\right)$. The field $\boldsymbol{v}$ approximates the effective displacement on the principal axes of the fibers and $\frac{\theta}{r_{\varepsilon}}$ is a local estimation of their angles of rotation with respect the same axes. For notational brevity, we set (8). We show that the effective problem associated with (1) is given by (9), where the set $\mathcal{D}$, which characterizes the boundary and regularity conditions satisfied by $\boldsymbol{v}^{\text {tuple }}$, is defined by (20) and

$$
\Phi\left(\boldsymbol{u}, \boldsymbol{v}^{\text {tuple }}\right)=\int_{\Omega} f\left(\boldsymbol{e}(\boldsymbol{u}) d x+\int_{\Omega} c^{f}(\boldsymbol{v}-\boldsymbol{u}, \theta) d x+\Phi_{f i b e r s}\left(\boldsymbol{v}^{\text {tuple }}\right) .\right.
$$

The second term of $\Phi$ represents a concentration of strain energy arising in a thin region of space enveloping the fibers. This extra contribution is generated by the emergence of a gap, characterized by $(\boldsymbol{v}-\boldsymbol{u}, \theta)$, between the effective displacements in the fibers and in the matrix. For $(\boldsymbol{a}, \alpha) \in \mathbb{R}^{3} \times \mathbb{R}$, the extended real $c^{f}(\boldsymbol{a}, \alpha)$ represents the limit of the sum of the images of the connected components of the cross section of the fibers under $\operatorname{cap}^{f}(., \widehat{\Omega} ; \boldsymbol{a}, \alpha)$ per unit surface, where, for any open subsets $U, V$ of $\mathbb{R}^{2}$ such that $U$ is bounded and $\bar{U} \subset V$, denoting by $\boldsymbol{x}_{U}$ the geometrical center of gravity of $U$, the application cap ${ }^{f}$ is defined by (11). Similar notions of capacity are considered in [2], [10]. Equivalently, $c^{f}(\boldsymbol{a}, \alpha)$ is given by (12). The computation of $c^{f}(\boldsymbol{a}, \alpha)$ requires a study of $\operatorname{cap}^{f}$ which reveals striking differences depending on the rate of growth $p$ of the function $f$. We focuse on the most interesting case $1<p<2$; the case $2 \leq p<+\infty$ is commented in Remark 1 . Then, the density $c^{f}$ is given by (13) in terms of the $p$-recession function $f^{\infty, p}$ of $f$ and of the parameter $\gamma^{(p)}$ defined by (14). We suppose (15), (16). The functional $\Phi_{\text {fibers }}$ in (2) represents the effective strain energy stored in the fibers. It depends on the order of magnitude of the parameters $k$ and $\kappa$ defined by (6), and is given by (17), where the $g_{1}^{\text {hom }}: \mathbb{R}^{2} \rightarrow \mathbb{R}$ is defined by (18) and $g_{2}^{\text {hom }}: \mathbb{R}^{4} \rightarrow \mathbb{R}$ is given by (19). If $0<k<+\infty$, the effective strain energy stored in the fibers involves both torsional and stretching strain energies whereas if $0<\kappa<+\infty$, it couples bending, torsional and stretching strain energies. In the other cases, it vanishes. An effective energy similar to that described by $\Phi_{\text {fibers }}$ in the case $0<k<+\infty$ is obtained in [1] in a quite different context of high contrast homogenization. The next theorem extends the results obtained in [4], [9] in the setting of linear isotropic elasticity.

Email address: michel.bellieud@univ-montp2.fr (Bellieud Michel). 
Theorem 0.1 Assume (3)-(16), let $\boldsymbol{u}_{\varepsilon}$ be the solution to (1) and let $\boldsymbol{v}_{\varepsilon}$ and $\theta_{\varepsilon}$ be the operators defined by (7). Then the sequence $\left(\boldsymbol{u}_{\varepsilon}\right)$ converges weakly in $W^{1, p}\left(\Omega ; \mathbb{R}^{3}\right)$ to $\boldsymbol{u}$, the sequence $\left(\boldsymbol{v}_{\varepsilon}\left(\boldsymbol{u}_{\varepsilon}\right), \theta_{\varepsilon}\left(\boldsymbol{u}_{\varepsilon}\right)\right)$ converges weakly in $L^{p}\left(\Omega ; \mathbb{R}^{3}\right) \times L^{p}(\Omega)$ to $(\boldsymbol{v}, \theta)$, and, if $0<\kappa<+\infty$, the sequence $\left.\left(\frac{1}{r_{\varepsilon}}\left(\boldsymbol{v}_{\varepsilon}\left(\boldsymbol{u}_{\varepsilon}\right)\right)_{3}, \frac{1}{r_{\varepsilon}} \theta\left(\boldsymbol{u}_{\varepsilon}\right)\right)\right)$ converges weakly in $\left(L^{p}(\Omega)\right)^{2}$ to $(w, \delta)$, where $(\boldsymbol{u}, \boldsymbol{v}, \theta)$ (respectively $(\boldsymbol{u}, \boldsymbol{v}, \theta, w, \delta)$ if $\left.0<\kappa<+\infty\right)$ is the unique solution of $(9)$.

Remark 1 (i) If $p=2$, the sequence $\left(\theta_{\varepsilon}\left(\boldsymbol{u}_{\varepsilon}\right)\right)$ converges to 0 in $L^{p}(\Omega)$. If the matrix is made up of linear isotropic material, namely if $f$ takes the form (21), then the effective problem is deduced from (9) by substituting (22) for (13) in (10). If in addition the fibers are made of a homogeneous isotropic material, then the torsion terms disappear. In this way, we recover the results established in [4], [9].

(ii) If $2<p<+\infty$, the sequence $\left(\boldsymbol{v}_{\varepsilon}\left(\boldsymbol{u}_{\varepsilon}\right), \theta_{\varepsilon}\left(\boldsymbol{u}_{\varepsilon}\right)\right)$ converges to 0 in $L^{p}\left(\Omega ; \mathbb{R}^{3}\right) \times L^{p}(\Omega)$. The effective problem is deduced from (9) by substituting (23) for (13) in (10).

The proof of Theorem 0.1 is based on $\Gamma$-convergence techniques [6]. For more details, we refer to [3].

\section{Introduction}

Soit $\Omega:=\widehat{\Omega} \times(0, L)$ un domaine borné cylindrique de $\mathbb{R}^{3}$. Etant donné un domaine borné Lipschitzien $S$ de $\mathbb{R}^{2}$ et une suite $\left(r_{\varepsilon}\right)$ de réels telle que

$$
0<r_{\varepsilon}<<\varepsilon
$$

on définit la distribution $\varepsilon$-périodique de "fibres" parallèles $T_{r_{\varepsilon}}$ en posant

$$
\begin{aligned}
& T_{r_{\varepsilon}}=S_{r_{\varepsilon}} \times(0, L), \quad S_{r_{\varepsilon}}=\bigcup_{i \in I_{\varepsilon}} S_{r_{\varepsilon}}^{i}, \quad S_{r_{\varepsilon}}^{i}=\varepsilon \boldsymbol{i}+r_{\varepsilon} S, \\
& I_{\varepsilon}:=\left\{i \in \mathbb{Z}^{2}, Y_{\varepsilon}^{i} \subset \widehat{\Omega}\right\}, \quad Y_{\varepsilon}^{i}:=\varepsilon\left(\{\boldsymbol{i}\}+\left[-\frac{1}{2}, \frac{1}{2}\left[^{2}\right) .\right.\right.
\end{aligned}
$$

Nous nous intéressons à l'homogénéisation du problème d'élasticité (1) lorsque, notant $\mathbb{S}^{3}$ l'ensemble des matrices symétriques réelles d'ordre 3 , les fonctions $f, g: \mathbb{S}^{3} \rightarrow \mathbb{R}$ sont strictement convexes et satisfont une condition de croissance d'ordre $p \in(1,+\infty)$ du type

$$
c|\boldsymbol{M}|^{p} \leq h(\boldsymbol{M}) \leq C\left(1+|\boldsymbol{M}|^{p}\right) \quad \forall \boldsymbol{M} \in \mathbb{S}^{3}, \quad h=f, g,
$$

et que $\left(k_{\varepsilon}\right)$ est une suite de réels positifs vérifiant

$$
\left.\left.\lim _{\varepsilon \rightarrow 0}\left(\frac{r_{\varepsilon}^{2}|S|}{\varepsilon^{2}} k_{\varepsilon}\right)^{\frac{1}{p}}=k \in\right] 0,+\infty\right], \quad \lim _{\varepsilon \rightarrow 0} r_{\varepsilon}\left(\frac{r_{\varepsilon}^{2}|S|}{\varepsilon^{2}} k_{\varepsilon}\right)^{\frac{1}{p}}=\kappa \in[0,+\infty] .
$$

Pour simplifier, on suppose que $g$ est positivement homogène de degré $p$. Le problème limite est exprimé en fonction de la limite $\boldsymbol{u}$ de la suite $\left(\boldsymbol{u}_{\varepsilon}\right)$ des solutions de (1), de la limite $(\boldsymbol{v}, \theta)$ de la suite $\left(\boldsymbol{v}_{\varepsilon}\left(\boldsymbol{u}_{\varepsilon}\right), \theta_{\varepsilon}\left(\boldsymbol{u}_{\varepsilon}\right)\right)$, où les opérateurs $\boldsymbol{v}_{\varepsilon}$ et $\theta_{\varepsilon}$ sont définis sur $W^{1, p}\left(\Omega ; \mathbb{R}^{3}\right)$, notant $\boldsymbol{x}_{S}$ le barycentre de $S$, par

$$
\begin{aligned}
\boldsymbol{v}_{\varepsilon}(\varphi)(x) & :=\sum_{i \in I_{\varepsilon}}\left(f_{S_{r_{\varepsilon}}^{i}} \varphi\left(s, x_{3}\right) d \mathcal{H}^{2}(s)\right) 1_{Y_{\varepsilon}^{i}}\left(x_{1}, x_{2}\right), \\
\theta_{\varepsilon}(\varphi)(x) & :=\sum_{i \in I_{\varepsilon}} \frac{\operatorname{diam} S}{2 f_{S}|\boldsymbol{y}|^{2} d y} f_{S_{r_{\varepsilon}}^{i}}\left(-\frac{y_{\varepsilon 2}}{r_{\varepsilon}} \varphi_{1}+\frac{y_{\varepsilon 1}}{r_{\varepsilon}} \varphi_{2}\right)\left(s, x_{3}\right) d \mathcal{H}^{2}(s) 1_{Y_{\varepsilon}^{i}}\left(x_{1}, x_{2}\right), \\
\boldsymbol{y}_{\varepsilon}\left(x_{1}, x_{2}\right) & :=\sum_{i \in I_{\varepsilon}}\left(\left(x_{1}, x_{2}\right)-\varepsilon\left(\left(i_{1}, i_{2}\right)+\boldsymbol{x}_{S}\right) 1_{Y_{\varepsilon}^{i}}\left(x_{1}, x_{2}\right)\right.
\end{aligned}
$$


et, lorsque $0<\kappa<+\infty$, de la limite $(w, \delta)$ de la suite $\left(\frac{1}{r_{\varepsilon}} \mathfrak{v}_{\varepsilon 3}\left(\boldsymbol{u}_{\varepsilon}\right), \frac{1}{r_{\varepsilon}} \theta_{\varepsilon}\left(\boldsymbol{u}_{\varepsilon}\right)\right)$. Ces quantités sont en fait l'éclatement (au sens de la notion introduite par D. Cionarescu-A. Damlamian- G. Griso dans [5]) des déplacements des lignes moyennes des fibres de section $S$. Le champ $\boldsymbol{v}$ représente le déplacement effectif sur l'axe principal des fibres et le champ $\frac{\theta}{r_{\varepsilon}}$ est une approximation de leur angle de rotation autour de cet axe. On note

$$
\boldsymbol{v}^{\text {tuple }}:= \begin{cases}(\boldsymbol{v}, \theta, w, \delta) & \text { si } 0<\kappa<+\infty \\ (\boldsymbol{v}, \theta) & \text { sinon }\end{cases}
$$

\section{2. Énoncé du résultat}

Nous montrons que le problème effectif s'écrit

$$
\left(\mathcal{P}^{\text {hom }}\right): \min _{\left(\boldsymbol{u}, \boldsymbol{v}^{\text {tuple }}\right) \in W_{b}^{1, p}(\Omega) \times \mathcal{D}} \Phi\left(\boldsymbol{u}, \boldsymbol{v}^{\text {tuple }}\right)-\int_{\Omega} \boldsymbol{f} \cdot \boldsymbol{u} d x,
$$

où $\mathcal{D}$ est défini par (20) et

$$
\Phi\left(\boldsymbol{u}, \boldsymbol{v}^{\text {tuple }}\right)=\int_{\Omega} f\left(\boldsymbol{e}(\boldsymbol{u}) d x+\int_{\Omega} c^{f}(\boldsymbol{v}-\boldsymbol{u}, \theta) d x+\Phi_{\text {fibers }}\left(\boldsymbol{v}^{\text {tuple }}\right) .\right.
$$

Le second terme de $\Phi$ décrit une concentration d'énergie élastique se produisant dans une portion de matrice qui entoure les fibres et dont le volume tend vers 0 lorsque $\varepsilon \rightarrow 0$. Ce phénomène résulte de la différence, caractérisée par $(\boldsymbol{v}-\boldsymbol{u}, \theta)$, entre les comportements asymptotiques du déplacement dans les fibres et dans la matrice. Pour tout $(\boldsymbol{a}, \alpha) \in \mathbb{R}^{3} \times \mathbb{R}$, le nombre $c^{f}(\boldsymbol{a}, \alpha)$ représente la limite de la somme

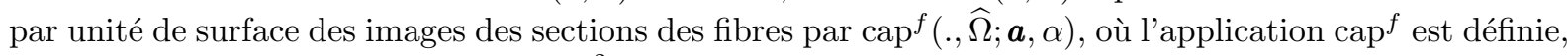
pour tout couple $(U, V)$ d'ouverts de $\mathbb{R}^{2}$ tel que $U$ soit borné et d'adhérence incluse dans $V$ par

$$
\operatorname{cap}^{f}(U, V ; \boldsymbol{a}, \alpha):=\inf \left\{\int_{V} f(\boldsymbol{e}(\boldsymbol{\psi})) d x, \begin{array}{l}
\boldsymbol{\psi} \in \mathcal{D}\left(V ; \mathbb{R}^{3}\right), \\
\boldsymbol{\psi}=\boldsymbol{a}+\frac{2}{\operatorname{diam} U} \alpha \boldsymbol{e}_{3} \wedge\left(\boldsymbol{x}-\boldsymbol{x}_{U}\right) \text { dans } S
\end{array}\right\} .
$$

Des notions de capacité similaires sont considérées dans [2], [10]. On obtient

$$
c^{f}(\boldsymbol{a}, \alpha)=\lim _{\varepsilon \rightarrow 0} \frac{1}{\varepsilon^{2}} \operatorname{cap}^{f}\left(r_{\varepsilon} S, \widehat{\Omega} ; \boldsymbol{a}, \alpha\right) \quad \forall(\boldsymbol{a}, \alpha) \in \mathbb{R}^{3} \times \mathbb{R} .
$$

Le calcul de $c^{f}(\boldsymbol{a}, \alpha)$ nécessite une étude de cap $^{f}$ qui révèle des différences importantes qui dépendent du choix du taux de croissance $p$ de la fonction $f$. Nous nous concentrons sur le cas $1<p<2$; le cas $2 \leq p<+\infty$ est commenté dans la remarque 1 . La fonction $c^{f}$ est alors donnée par

$$
c^{f}(\boldsymbol{a}, \alpha)=\gamma^{(p)} \operatorname{cap}^{f^{\infty, p}}\left(S, \mathbb{R}^{2} ; \boldsymbol{a}, \alpha\right) \quad \forall(\boldsymbol{a}, \alpha) \in \mathbb{R}^{3} \times \mathbb{R},
$$

en fonction de la fonction de $p$-récession $f^{\infty, p}$ de $f$ et du paramètre $\gamma^{(p)}$ définis par

$$
f^{\infty, p}(\xi):=\limsup _{t \rightarrow+\infty} \frac{f(t \xi)}{t^{p}}, \quad \gamma^{(p)}:=\lim _{\varepsilon \rightarrow 0} \frac{r_{\varepsilon}^{2-p}}{\varepsilon^{2}} .
$$

Nous supposons que

$$
\exists \beta>0, \exists \delta \in(0, p), \quad\left|f(\boldsymbol{M})-f^{\infty, p}(\boldsymbol{M})\right| \leq \beta\left(1+|\boldsymbol{M}|^{p-\delta}\right) \quad \forall \boldsymbol{M} \in \mathbb{S}^{3},
$$

et, pour simplifier, que 


$$
0<\gamma^{(p)} \leq+\infty
$$

La fonctionnelle $\Phi_{\text {fibers }}$, présente dans (10), décrit l'énergie élastique effective stockée dans les fibres. Sa forme dépend de l'ordre de grandeur des paramètres $k$ et $\kappa$ définis par (6). On obtient

$$
\Phi_{\text {fibers }}\left(\boldsymbol{v}^{\text {tuple }}\right)= \begin{cases}\int_{\Omega} g_{1}^{\text {hom }}\left(\frac{\partial v_{3}}{\partial x_{3}}, \frac{\partial \theta}{\partial x_{3}}\right) d x & \text { si } 0<k<+\infty, \\ \int_{\Omega} g_{2}^{\text {hom }}\left(\frac{\partial^{2} v_{1}}{\partial x_{3}^{2}}, \frac{\partial^{2} v_{2}}{\partial x_{3}^{2}}, \frac{\partial w}{\partial x_{3}}, \frac{\partial \delta}{\partial x_{3}}\right) d x & \text { si } 0<\kappa<+\infty, \\ 0 & \text { sinon },\end{cases}
$$

où la fonction $g_{1}^{\text {hom }}: \mathbb{R}^{2} \rightarrow \mathbb{R}$ est définie par

$$
\begin{aligned}
& g_{1}^{\text {hom }}(a, \beta):=\inf _{\boldsymbol{q} \in C^{\infty}\left(\bar{S} ; \mathbb{R}^{3}\right)}\left\{f_{S} g(k \mathfrak{\mathbb { H }}(\boldsymbol{q}, a, \beta)) d y\right\}, \\
& \mathbb{\mathfrak { L }}(q, a, \beta):=\boldsymbol{e}(\boldsymbol{q})+\frac{2}{\operatorname{diam} S} \beta\left(\begin{array}{ccc}
0 & 0 & -y_{2} \\
0 & 0 & y_{1} \\
-y_{2} & y_{1} & 0
\end{array}\right)+a\left(\begin{array}{lll}
0 & 0 & 0 \\
0 & 0 & 0 \\
0 & 0 & 1
\end{array}\right),
\end{aligned}
$$

et $g_{2}^{\text {hom }}: \mathbb{R}^{4} \rightarrow \mathbb{R}$ est donnée par

$$
\begin{aligned}
& g_{2}^{h o m}\left(w_{1}, w_{2}, a, \beta\right):=\inf _{\boldsymbol{q} \in C^{\infty}\left(\bar{S} ; \mathbb{R}^{3}\right)}\left\{f_{S} g\left(\kappa \mathfrak{J}\left(\boldsymbol{q}, w_{1}, w_{2}, a, \beta\right)\right) d y\right\}, \\
& \mathfrak{J}\left(\boldsymbol{q}, w_{1}, w_{2}, a, \beta\right):=\mathfrak{\mathbb { I }}(\boldsymbol{q}, a, \beta)-\left(\sum_{\alpha=1}^{2} w_{\alpha} y_{\alpha}\right)\left(\begin{array}{lll}
0 & 0 & 0 \\
0 & 0 & 0 \\
0 & 0 & 1
\end{array}\right) .
\end{aligned}
$$

L'énergie élastique effective stockée dans les fibres est un couplage d'énergies de torsion et d'extension si $0<k<+\infty$, et de flexion, torsion et extension si $0<\kappa<+\infty$. Dans les autres cas, elle s'annule. Une énergie de forme similaire à celle du cas $0<k<+\infty$ est obtenue dans [1] pour des composites de fibres fortement contrastés de nature très différente. L'ensemble $\mathcal{D}$ qui apparaît dans (9) décrit les conditions aux limites et la régularité satisfaites par $\left(\boldsymbol{v}^{\text {tuple }}\right)$. Il est défini par

$$
\mathcal{D}:=\left\{\begin{array}{cc}
\left\{(\boldsymbol{v}, \theta), \boldsymbol{v} \in L^{p}\left(\Omega ; \mathbb{R}^{3}\right), \quad\left(v_{3}, \theta\right) \in\left(L^{p}\left(\widehat{\Omega} ; W^{1, p}(0, L)\right)\right)^{2},\right. \\
\left.v_{3}=\theta=0 \text { sur } \widehat{\Omega} \times\{0\}\right\} & \text { si } 0<k<+\infty, \\
\left\{(\boldsymbol{v}, \theta), \boldsymbol{v} \in L^{p}\left(\Omega ; \mathbb{R}^{3}\right), v_{3}=\theta=0\right\} & \text { si }(k, \kappa)=(+\infty, 0), \\
\left\{(\boldsymbol{v}, \theta, w, \delta), \boldsymbol{v} \in L^{p}\left(\widehat{\Omega} ; W^{2, p}\left(0, L ; \mathbb{R}^{3}\right)\right), w, \delta \in L^{p}\left(\widehat{\Omega} ; W^{1, p}(0, L)\right),\right. & \\
\left.v_{3}=\theta=0, w=\delta=v_{\alpha}=\frac{\partial v_{\alpha}}{\partial x_{3}}=0 \text { sur } \widehat{\Omega} \times\{0\}(\alpha \in\{1,2\})\right\} & \text { si } 0<\kappa<+\infty, \\
\{(\boldsymbol{v}, \theta), \boldsymbol{v}=\theta=0\} & \text { si } k=\kappa=+\infty .
\end{array}\right.
$$

Le théorème suivant généralise les résultats obtenus dans [4], [9] dans le contexte de l'élasticité linéaire isotrope.

Theorem 2.1 Supposons (3)-(16), soit $\boldsymbol{u}_{\varepsilon}$ la solution de (1) et soient $\boldsymbol{v}_{\varepsilon}$ et $\theta_{\varepsilon}$ les opérateurs définis par (7). Alors la suite $\left(\boldsymbol{u}_{\varepsilon}\right)$ converge faiblement dans $W^{1, p}\left(\Omega ; \mathbb{R}^{3}\right)$ vers $\boldsymbol{u}$, la suite $\left(\boldsymbol{v}_{\varepsilon}\left(\boldsymbol{u}_{\varepsilon}\right), \theta_{\varepsilon}\left(\boldsymbol{u}_{\varepsilon}\right)\right)$ converge 
faiblement dans $L^{p}\left(\Omega ; \mathbb{R}^{3}\right) \times L^{p}(\Omega)$ vers $(\boldsymbol{v}, \theta)$, et, si $0<\kappa<+\infty$, la suite $\left(\frac{1}{r_{\varepsilon}}\left(\boldsymbol{v}_{\varepsilon}\left(\boldsymbol{u}_{\varepsilon}\right)\right)_{3}, \frac{1}{r_{\varepsilon}} \theta\left(\boldsymbol{u}_{\varepsilon}\right)\right)$ converge faiblement dans $\left(L^{p}(\Omega)\right)^{2}$ vers $(w, \delta)$, où $(\boldsymbol{u}, \boldsymbol{v}, \theta)$ (respectivement $(\boldsymbol{u}, \boldsymbol{v}, \theta, w, \delta)$ si $0<\kappa<+\infty$ ) est l'unique solution de (9).

Remarque 1 (i) Si $p=2$, alors la suite $\left(\theta_{\varepsilon}\left(\boldsymbol{u}_{\varepsilon}\right)\right)$ converge fortement vers 0 dans $L^{p}(\Omega)$. Lorsque $f$ est définie par

$$
f(\boldsymbol{M})=\frac{\lambda_{0}}{2}(\operatorname{tr} \boldsymbol{M})^{2}+\mu_{0} \boldsymbol{M}: \boldsymbol{M} \quad \forall \boldsymbol{M} \in \mathbb{S}^{3}, \quad \mu_{0}>0, \quad \lambda_{0} \geq 0,
$$

le problème effectif se déduit de (9) en remplaçant (13) par (22) dans (10), où

$$
c^{f}(\boldsymbol{a}, \alpha)=\left\{\begin{array}{ll}
\mu_{0} \pi \gamma^{(2)}\left(2 \frac{\lambda_{0}+2 \mu_{0}}{\lambda_{0}+3 \mu_{0}}\left(a_{1}+a_{2}\right)+a_{3}\right) & \text { si } \alpha=0, \\
+\infty & \text { sinon, }
\end{array} \quad \gamma^{(2)}:=\lim _{\varepsilon \rightarrow 0} \frac{1}{\varepsilon^{2}\left|\log r_{\varepsilon}\right|} .\right.
$$

Si de plus les fibres sont faites d'un matériau homogène isotrope, alors les termes de torsion disparaissent. On retrouve ainsi les résultats obtenus dans [4], [9].

(ii) Si $2<p<+\infty$, la suite $\left(\boldsymbol{v}_{\varepsilon}\left(\boldsymbol{u}_{\varepsilon}\right), \theta_{\varepsilon}\left(\boldsymbol{u}_{\varepsilon}\right)\right)$ converge fortement vers $(\boldsymbol{u}, 0)$ dans $L^{p}\left(\Omega ; \mathbb{R}^{3}\right) \times L^{p}(\Omega)$. Le problème effectif se déduit alors de (9) en remplaçant (13) par (23) dans (10), où

$$
c^{f}(\boldsymbol{a}, \alpha)=\left\{\begin{array}{ll}
0 & \text { si } \boldsymbol{a}=0 \\
+\infty & \text { sinon }
\end{array} \text { and } \alpha=0,\right.
$$

La preuve du théorème 1.1 est basée sur des techniques de $\Gamma$-convergence [6]. Pour plus de détails, on se réfèrera à $[3]$.

\section{Références}

[1] M. Bellieud : Torsion effects in elastic composites with high contrast. SIAM J. Math. Anal. 41(6) (2010), pp. 2514-2553.

[2] M. Bellieud : A notion of capacity related to elasticity. Applications to homogenization. Arch. Ration. Mech. Anal., vol. 203, no1, (2012), pp. 137-187.

[3] M. Bellieud : Capacitary problems in elasticity with torsion effects. Preprint (2011).

[4] M. Bellieud, I. Gruais : Homogenization of an elastic material reinforced by very stiff or heavy fibers. Non local effects. Memory effects. J. Math. Pures Appl. 84, (2005) pp. 55-96.

[5] D. Cioranescu, A. Damlamian, G. Griso : The periodic unfolding method in homogenization. SIAM J. Math. Anal., 40 (4) (2008), pp. 1585-1620.

[6] G. Dal Maso : An Introduction to Г-Convergence. Birkhäuser (1993).

[7] A. Friaâ : La loi de Norton-Hoff généralisée en plasticité et viscoplasticité. Thèse de Doctorat d'état, Paris VI (1979).

[8] R.W. Ogden, Large deformation isotropic elasticity. On the correlation of theory and experiment for incompressible rubberlike solids, Proceedings of the Royal Society of London, Serie A, Mathematical and Physical Sciences, Vol. 326, No. 1567 (1972), pp. 565-584.

[9] C. Pideri, P. Seppecher, A second gradient material resulting from the homogenization of an heterogeneous linear elastic medium, Contin. Mech. Thermodyn., 9 (1997), pp. 241-257.

[10] P. Villaggio : The main elastic capacities of a spheroid, Arch. Ration. Mech. Anal. 92, n 4 (1986) pp. 337-353. 